\title{
BIOMECHANICS OF MALLET FINGER A CASE REPORT
}

\author{
Habib MA ${ }^{1}$, Haque $\mathrm{ME}^{2}$
}

\section{Introduction}

Mallet finger (also called drop or baseball finger) is a deformity of the finger caused by detachment of the extensor mechanism from the base of the distal phalanx, either directly or in association with a fracture ${ }^{1,2}$. The commonest cause of this injury is a violent flexion or laceration of the dorsum of the finger at the level of the distal inter-phalangeal (DIP) joint. It is most commonly seen when the finger is struck on the tip or hit against a solid object resulting in rupture of the extensor tendon or avulsion of the tendon with or without a small fragment of bone from the insertion. Mallet finger is the most common closed tendon injury that is seen in athletes; this injury is also common in non-athletes from relatively minor trauma or even from household tasks like bed making. The effect results in continuous flexion and inability to fully extend the last joint of the finger. The resultant deformity occurs by unopposed flexion of the distal phalanx $x^{1,3}$.

Anatomy of the extensor mechanism of fingers shows that extrinsic and intrinsic extensor tendons fuse together to anchor in the base of distal phalanx. So injury to this line of action causes failure of extensor activity of DIP joint ${ }^{4}$.

Aim of the present study is to report a case of mallet finger and exploration of anatomical background of such deformity to have better confidence in management of such cases.

\section{Case Report}

A 44 years old soldier of Bangladesh Rifles (BDR) reported to Medical Inspection (MI) Room of Sector Head Quarters, BDR Khagrachari on 31 January 2008 with deformity of left little finger and pain. The patient received an injury during playing volley ball, 14 days before reporting. He stated that, he was pushing ball from below keeping the palm partially open. Immediately after injury he felt severe pain and finger was deformed. He did not report to doctor and went on leave on the following day. Pain reduced on self medication, but deformity persisted.

At the time of reporting to doctor the patient was not feeling any problem in working with left hand except little pain in and restricted movement of left little finger. On examination the left little finger was observed hyperextended at proximal inter-phalangeal (PIP) joint and flexed at DIP joint (Fig-1). There was no active movement at DIP joint. Movements at PIP and metacarpo- phalangeal (MP) joints were normal. Cutaneous sensation was normal in fingers and there was no wasting of thenar and hypothenar muscles. The X-ray of left little finger showed that there was an avulsion fracture of part of base of distal phalanx (Fig - $2 \& 3$ ) of left little finger.

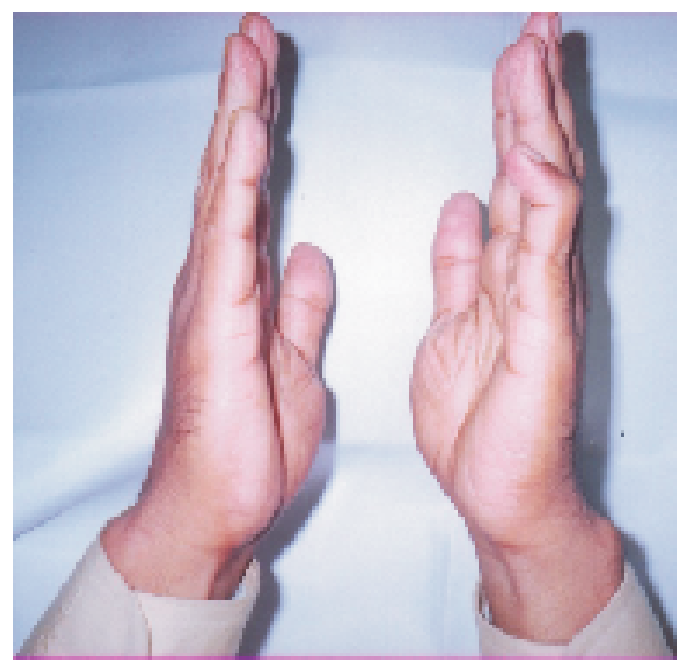

Fig-1: Mallet left little finger.

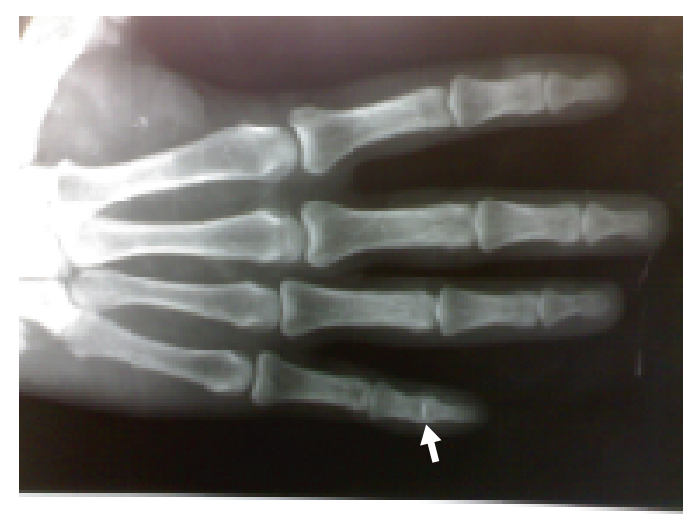

Fig-2: No joint space in DIP joint of little finger.

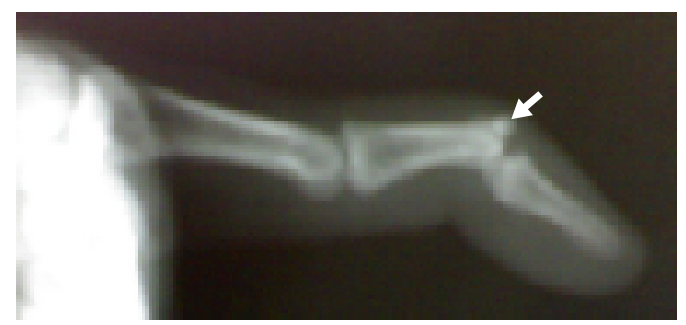

Fig-3: Fracture of base of the distal phalanx (arrow). Fractured bone piece is pulled away.

1. Lt Col Md Ahsan Habib MBBS, M Phil, Head of the Department, Anatomy, AFMC. 2. Dr Molla Ershadul Haque MBBS, MS Ortho, Consultant Orthopaedic Surgeon, BDR Hospital, Dhaka. 


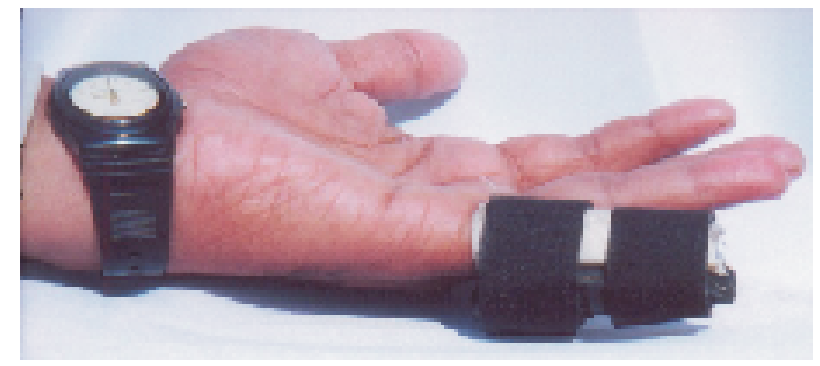

Fig- 4: Finger with splint.

he patient though reported late (14 days after) was treated conservatively. A locally made external finger splint (Fig- 4) was applied and was kept in situ for 8 weeks ${ }^{5,6}$. The fractured piece united with little deformity of bone (Fig- 5). Functional activity was restored by physiotherapy.
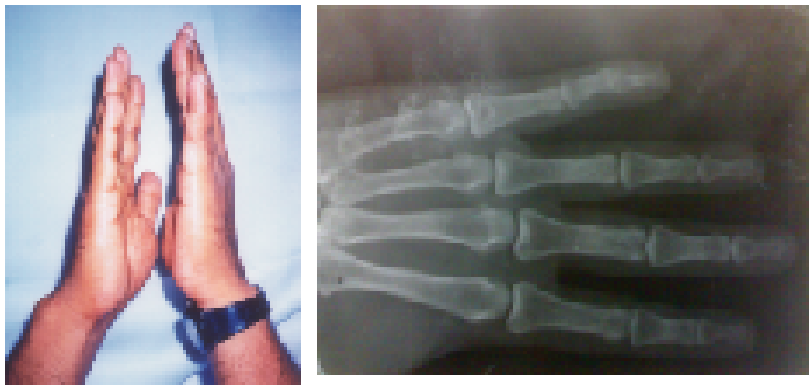

Fig-5: Photograph and X-ray after 8 weeks of splinting show union of bone with little deformity.

\section{Joints of Hand}

Each hand contains eight carpal, five metacarpal and fourteen phalanges (Fig-6). All the joints between them are synovial allowing varying degree of movements. Inter-carpal joints are plane synovial joints and allow limited movement. Carpo-metacarpal joint of thumb is sellar variety and allow multiaxial movements. On the contrary other four carpo-metacarpal joints are plane variety (some times considered modified sellar) and

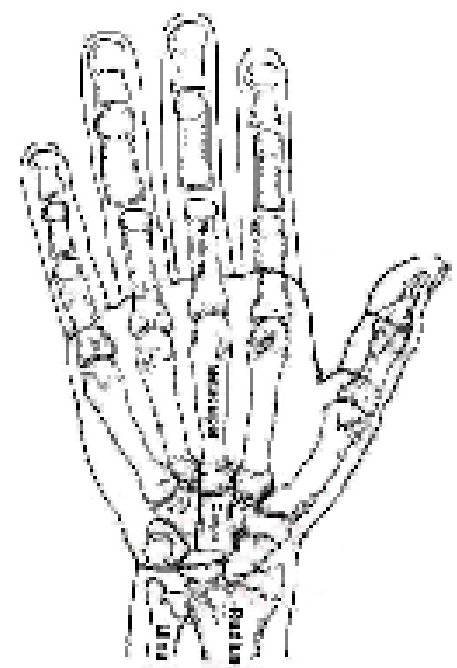

Fig-6: Bones and joints of hand. movement is marked restricted compared to that of thumb. Second to fifth metacarpal bases make joint with trapezoid \& trapezium, capitate \& trapezoid, capitate \& hamet and hamet respectively. There are also joints between bases of metacarpals and are plane allowing limited movement. Metacarpal heads form joint with bases of proximal phalanges. Joints are ellipsoid and allow wide range of movements. Inter-phalangeal joints are uniaxial hinge joints and range of movement is relatively more proximally ${ }^{4}$.

\section{Extensor of Finger}

Main bulk of extensor digitorum, extensor digiti minimi and extensor indicis muscles are present in extensor compartment of forearm (extrinsic muscle). They send narrow tendons to fingers which together with interosseous and lumbrical muscles (intrinsic muscle) tendon form dorsal digital or extensor expansions ${ }^{4}$. Extensor expansions are triangular fibrous expansions present on dorsum of each finger. Base of triangle is proximal and wraps around the dorsum and collateral aspects of metacarpo-phalangeal joint (Fig-7). Extensor digitorum muscle divides into four tendons and each of them proceeds to one finger except thumb. A tendon of extensor digitorum blends with the expansion along its central axis and is separated from metacarpo-phalangeal joint by a small bursa ${ }^{4}$. Margins of these expansions are thickened by tendons of the lumbrical and interosseous tendons. Abductor digiti minimi forms thickened margin

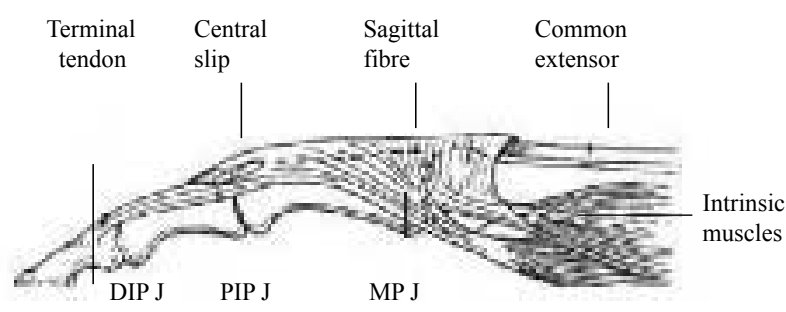

Fig-7: Extensor expansion of finger.

of expansion of little finger. Extensor expansion is very thin between tendons. Each of the extensor digitorum tendons split into axial part and two collateral slips, near proximal inter-phalangeal joint. Axial component receives some fibres from lumbrical and interosseous tendons and anchors into base of middle phalanx. Collateral slips join corresponding thick border and finally two borders unite and attach to dorsal aspect of base of distal phalanx. Detail study also shows that extensor expansions have attachments to the metacarpo-phalangeal joint palmar plate through the sagittal bands and tenuous insertions on the proximal phalanx ${ }^{4}$.

Extensor digitorum extends MP, PIP and DIP joints of medial four fingers. Extensor digiti minimi and extensor indicis do the same job on little and index fingers respectively. Lumbrical and interosseous muscles flex MP joints and extend IP joints of middle three fingers ${ }^{4}$. 
The terminal extensor tendon is a thin flat structure measuring approximately $1 \mathrm{~mm}$ thick and 4-5 mm wide. It inserts onto the dorsal lip of the distal phalanx $x^{7}$. Due to a less protected anatomical location, extensor tendon injuries are more frequently encountered than flexor tendon injuries ${ }^{1}$. Usually a sudden, forceful flexion of the DIP joint in an extended digit results in rupture of the extensor tendon or avulsion of the tendon often with a bony fragment from its insertion in the distal phalanx. This leads to mallet finger ${ }^{1,2}$. A mallet finger may be open but it is more often closed. Unopposed action of flexor of DIP cause continuous flexion of that joint and reaction contraction of central band of extensor expansion cause hyperextension of the PIP joint (swan neck deformity). There are four types of mallet finger injuries ${ }^{1,2}$ :

Type I: Closed with or without avulsion fracture

Type II: Laceration at or proximal to the DIP joint with loss of tendon continuity

Type III: Deep abrasion with loss of skin, subcutaneous cover and tendon substance

Type IV:

(A) Trans-epiphyseal plate fracture in children;

(B) Hyperextension injury with fracture of the articular surface of $20-50 \%$; and

(C) Hyperextension injury with fracture of the articular surface usually $>50 \%$ and with early or late palmar subluxation of the distal phalanx.

\section{Discussion}

Extensor expansion of little finger is similar to other fingers. Central band is formed by medial most split of extensor digitorum and extensor digiti minimi. Lateral band is formed by fourth palmar interosseous and fourth lumbrical muscle. Medial band is abductor digiti minimi tendon. Central tendon causes extensions of all the joints of little finger. Fourth palmar interosseous and fourth lumbrical muscles cause adduction and flexion of MCP joint and extension of IP joints of little finger. Abductor digiti minimi cause abduction and flexion of MCP joint and extension of IP joints. A mallet finger is a flexion deformity of the DIP joint. It is caused by a disruption of the extensor mechanism near its insertion into the distal phalanx $x^{1,2,4}$.

In reported case, sudden forceful flexion of the DIP joint in an extended little finger caused by volley ball resulted avulsion of the tendon with a bony fragment from base of the distal phalanx. This caused pain and flexion deformity of DIP joint immediately. As the patient reported 2 weeks after injury, reaction contraction of central slip of extensor expansion became prominent and that lead to hyper-extension of PIP joint (Fig-1). Investigation (X-ray) revealed that the reported patient developed type I mallet left little finger as per standard classification ${ }^{1,2}$.

Simpson in a study observed that of 851 patients reported to Edinburgh Orthopaedic Trauma Unit with an acute sporting injury $2 \%$ had either a soft tissue or bony mallet deformity $^{5}$.

The problem with a mallet deformity is that many patients neglect them as a "sprained finger". The specific difference between a sprain and a mallet finger is that latter is associated with a rupture of the tendon that extends the tip of finger or DIP joint. With the tendon rupture the first thing to happen is, one cannot actively extend the finger. One of the classic findings is the ability to passively extend the joint, but a loss of active extension and tenderness over the back of the joint ${ }^{3,7}$. A far less common finger injury is a "jammed finger." This results from an axial load to the finger. The joint surfaces of each bone "jam" together, causing a joint surface injury. This often results in swelling and loss of motion. X-ray usually does not show any sign of trauma and the joint does not exhibit instability ${ }^{5}$.

A clinically important fibro-osseous tunnel lies within the proximal part of the hypothenar eminence called Guyon's canal. It is bounded by the pisiform and hook of the hamate and is covered proximally by the palmar carpal ligament and distally by the short palmar muscle. The canal transmits terminal part of ulnar nerve and the ulnar artery. Within the canal, the ulnar nerve divides into superficial sensory and deep motor branches. The superficial branch on exit from the canal supplies a small branch to the palmaris brevis muscle and terminal branches provide sensation to the little finger and the ulnar half of the ring finger. The deep motor branch along with the ulnar artery takes an acute lateral turn about the hook of the hamate. This is the most vulnerable site for compression of the deep motor branch of the ulnar nerve. The deep motor branch innervates hypothenar muscles, $3 \mathrm{rd}$ and 4 th lumbricals and all interosse $\mathrm{i}^{4,8}$. Of course ulnar nerve may be compressed anywhere along the course of the Guyon's canal causing only motor or only sensory abnormalities or loss of both. Claw-finger deformity of the little finger, abductor digiti minimi atrophy without atrophy of the first dorsal interosseous muscle and hypoaesthesia on the ulnar side of the little finger followed by trauma to palm may be the presentation of ulnar nerve injury in Guyon's canal ${ }^{8,9}$.

Trauma from sports or from any other causes may lead to mallet finger deformity, sprained finger, jammed finger and ulnar nerve injury especially in Guyon's canal ${ }^{5,8}$. Careful examination and very simple investigation (Xray) can confirm diagnosis.

Type I mallet finger injury is better treated conservatively. Splinting of DIP joint in extension for 6 to 8 weeks yield good result ${ }^{1,5}$. Early immobilization shows better result ${ }^{6}$. A few days delay in initiation of treatment does not affect significantly the DIP joint movement or extension $\operatorname{lag}^{10}$. Due to delay in reporting, finger of reported patient was immobilized after 14 days of inflecting injury. The patient recovered with little deformity and physiotherapy could bring functional state back completely.

\section{Conclusion}

Mallet injuries are best treated using closed, nonoperative techniques ${ }^{6}$. Many of the Armed Forces personnel after induction start playing games like volley 
ball, basket ball etc. Because of inexperience in this field, they are prone to reported type of injury leading to mallet finger. Simple immobilization for 6 to 8 weeks yields complete recovery ${ }^{5,6}$. The patient may not initially seek treatment due to little functional deficit. A mallet deformity can lead to a swan-neck deformity if not treated ${ }^{2}$. So root level motivation for awareness is the aim of this publication.

\section{References}

1. Bendre A A, Hartigan B J, Kalainov D M. Mallet finger. Journal of the American Academy of Orthopaedic Surgeons 2005; 113 (5): 336-344.

2. Mauffrey C. Mallet finger - A review. The Internet Journal of Orthopaedic Surgery 2006; 3(1) : 1-5.

3. Peterson JJ, Bancroft LW. Injuries of the fingers and thumb in the athlete. Clinical Sports Medicine 2006 July; 25 (3) : 527 - 542.

4. Williams PL, Bannister LH, Berry MM, et al. Gray's Anatomy. 38th edn. Great Britain : Churchill Livingstone; 1995. p.646 - 662 \& p.858 - 863.
5. Simpson D, McQueen MM, Kumar P. Mallet deformity in sport. British Journal of Hand Surgery 2001 February; 26(1):32-33.

6. Jabecki J, Syrko M. Zone 1 extensor tendon lesions: current treatment methods and a review of literature. Orthop Traumatol Rehabil 2007 Jan-Feb; 9(1) : 52-62.

7. Ketchum LD, Thompson D, Pocock G, Wallingford D. A clinical study of forces generated by the intrinsic muscles of the index finger and the extrinsic flexor and extensor muscles of the hand. J Hand Surg [Am] 1978 Nov; 3(6): 571-8.

8. Aguiar PH, Bor-Seng-Shu E, Gomes-Pinto F, et al. Surgical management of Guyon,s canal syndrome. A ulnar nerve entrapment at wrist. Arquivos de Neuro-Pesiquiatria 2001 March ; 59 (1) : 1-11.

9. Rengachary SS, Arjunan K. Compression of the ulnar nerve in Guyon's canal by a soft tissue cell tumour. Neurosurgery 1981; $8: 400-405$.

10. Okafor B, Mbubaeqbu C, Munshi I, Williams DJ. Mallet deformity of finger, five year follow-up of conservative treatment. J Bone Joint Surg (Br) 1997 July;79 (4) : 544-7. 